\title{
Intrinsic and extrinsic nature of the giant piezoelectric effect in the initial poling of PMN-PT
}

\author{
A. G. A. Nisbet* and F. Fabrizi \\ Diamond Light Source, Didcot OX11 ODE, United Kingdom \\ S. C. Vecchini, M. Stewart, and M. G. Cain \\ National Physical Laboratory, Teddington TW11 OLW, United Kingdom \\ T. Hase \\ University of Warwick, Coventry CV4 7AL, United Kingdom \\ P. Finkel \\ U.S. Naval Research Laboratory, Washington, DC 20375, USA \\ S. Grover and R. Grau-Crespo $\odot$ \\ Department of Chemistry, University of Reading, Whiteknights, Reading RG6 6AD, United Kingdom \\ S. P. Collins \\ Diamond Light Source, Didcot OX11 ODE, United Kingdom
}

(Received 6 November 2020; revised 14 September 2021; accepted 19 November 2021; published 17 December 2021)

\begin{abstract}
Demonstrating both the intrinsic and extrinsic nature of the giant piezoelectric effect (GPE) in complex solid solutions, near the morphotropic phase boundary, has been extremely challenging until now, because such materials exhibit multiple phases on the order of tens of microns across, meaning important information is lost due to averaging when using established high resolution diffraction techniques to extract three dimensional structural information. We have used a different approach proposed by Nisbet et al. [Acta Crystallogr. Sect. A 71, 20 (2015)], which has been adapted to differentiate between spatially adjacent phases and simultaneously track the evolution of those phases in response to electric fields. As a result, we have identified three environment specific GPEs. The first of these is a GPE which is an order of magnitude greater than previously reported for a given change in field. This is observed during a tetragonal-monoclinic transition in a multiphasic environment. A secondary, large GPE is observed in the neighboring, nontransitioning, monoclinic phase due to stress biasing, and a more typical GPE is observed when the system becomes monophasic. Our results demonstrate the simultaneous and complex interplay of intrinsic and extrinsic factors contributing to the GPE which is likely to have implications for device manufacture and miniaturization.
\end{abstract}

DOI: 10.1103/PhysRevMaterials.5.L120601

Faster, more energy efficient transistors in the form of piezoelectric effect transistors (PETs) are potentially on the horizon. PET devices can be created by combining piezoelectric and piezoresistive materials to make logic switches. A piezoelectric material exhibiting the giant piezoelectric effect (GPE) is used to compress a piezoresistive material driving it through a phase transition to become a conductor. Such PET devices could achieve 8-GHz clock frequencies with line voltages as low as $115 \mathrm{mV}$, reducing power consumption by two orders of magnitude compared with current complementary

\footnotetext{
*gareth.nisbet@diamond.ac.uk

Published by the American Physical Society under the terms of the Creative Commons Attribution 4.0 International license. Further distribution of this work must maintain attribution to the author $(s)$ and the published article's title, journal citation, and DOI.
}

metal-oxide semiconductor technology [1]. The significance of this is underscored by the fact the world's information technology infrastructure now draws an estimated $10 \%$ of the world's electrical power [2]. These new devices would be a leap forward in greener technology. However, before these materials can form the basis of this new technology, their underpinning properties need to be understood, predictable, and controllable.

Currently there are two dominant models for the mechanism of the GPE. One assertion is that the phenomenon arises from a continuous rotation of the polarization vector which manifests as a distortion in the unit cell. Fu et al. [3] proposed a methodology in which the phase stability is studied using the first-principles linearized augmented plane wave method in conjunction with the local density-functional approximation. They calculated the internal energies for several polarization directions and established a likely polarization pathway beginning with a rhombohedral phase transitioning 
to a tetragonal phase via a monoclinic bridge. It is these polymorphic transitions, they suggest, which are responsible for the GPE. In fact, they conclude that the GPE can be understood entirely in terms of polarization rotation and efforts to design better piezoelectric materials should focus on their intrinsic structure. Noheda et al. [4,5] also observed a monoclinic phase using high energy $\mathrm{x}$-ray diffraction and pointed out that this is consistent with the polarization pathway proposed by $\mathrm{Fu}$ et al. However the monoclinic phase poses a challenge to Devenshire theory which provides a phenomenological description of many cubic perovskite ferroelectrics via a sixth order expansion of the free energy in the polar order parameter [6]. While this is not insurmountable and can be described by an eighth order expansion, the necessity for which they attribute to an unusually anharmonic free-energy surface [6], Jin et al. [7] propose an alternative explanation which is the second of the two dominant models. They extend martensite theory to ferroelectric systems and argue that the monoclinic phase is a homogenized mixed state of twin-related tetragonal microdomains and the GPE arises from a change in ratio of population density. Calculations by Rossetti et al. [8] using a low-order Landau expansion satisfying the Gibbs phase rule have suggested that, in the narrow range of the morphotropic phase boundary, the coexistence of rhombohedral and tetragonal symmetries is thermodynamically favorable and this coexistence is likely to occur as nanodomains. Schönau et al. [9] using a plethora of techniques such as high resolution $\mathrm{x}$-ray powder diffraction (XRD), convergent beam electron diffraction (CBED), and transmission electron microscopy (TEM) conclude in favor of the microdomain adaptive phase model.

Despite the obvious technological importance of such materials, demonstrated by the sheer volume of publications, attempting to explain the dominant mechanisms responsible for the GPE near the morphotropic phase boundary, a clear understanding of the relationship between the physical properties and the underlying crystal structure has, thus far, remained elusive. This is partly because of the intractability of such complex systems from a theoretical perspective, inconsistency between samples, or limitations in the experimental methods. A scale referenced probe capable of tracking the evolution of phases as a function of time, temperature, in situ electric field, stress, and other technologically important parameters while sampling a fixed sample volume without averaging is required.

Rurtherford and Andrade observed divergent beam diffraction lines as early as 1914. Numerous observations of similar phenomena were subsequently reported by Seemann (1916), Fujiwara (1928), and Kossel (1935) [10-12]. However, it was not until 1947 that the exceptional resolution and elegant simplicity of using these lines for lattice parameter determination were demonstrated by Lonsdale [13]. She noticed the occurrence of triple intersections among the diffraction lines (points where diffraction cones from three different planes coincide). Some of these, she termed "geometrically inevitable" arising from coplanar reflections, the others she termed "accidental" which occur uniquely for a given energy and set of lattice parameters. This meant that many of the uncertainties associated with the experimental geometry could be eliminated, facilitating very precise lattice determination in single crystals.
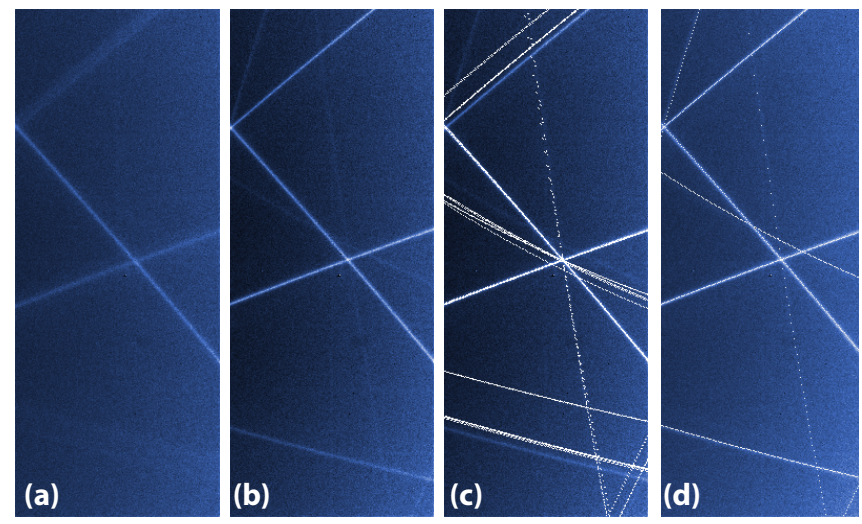

FIG. 1. (a) Image of DMS lines from unpoled PMN-PT 32\%, (b) poled PMN-PT 32\%. (c) Overlay of triple intersection solutions for cubic, rhombohedral, orthorhombic, and tetragonal constraints, (d) solution using monoclinic constraints for two triple intersections, comprised of the $\overline{2} \overline{1} 0, \overline{3} 03, \overline{1} 1 \overline{1}$ reflections at $7.301 \mathrm{keV}$ and the $\overline{2} \overline{1} 0, \overline{3} 03,020$ reflections at $7.309 \mathrm{keV}$.

However, the accidental nature of the triple intersections meant the technique was only suitable for certain source/sample pairings significantly reducing the applicability of the technique. Similarly, Kossel lines, which are produced from characteristic fluorescence, suffer from the same limitation. A scattering technique referred to as diffuse multiple scattering (DMS) [14] has been developed which can exploit the geometrical tricks discovered by Lonsdale but which can also exploit the tunable energy and low divergence of synchrotron sources, providing spacial resolution of a few microns. The so called accidental intersections can now be actively tuned by selecting the appropriate incident energy. Until now the technique has only been demonstrated on single crystals, but we show its real utility is with complex systems such as solid solutions.

As DMS arises from multiple internal sources, all crystalline phases and reflections contribute simultaneously. Thus, phase identification and lattice parameter determination better than $10^{-4} \AA$ within a well defined fixed volume is possible without moving the sample, avoiding the averaging which can occur due to instrumental sphere errors. This makes DMS ideal for exploring intrinsic and extrinsic properties in functional materials.

The sample $\left[\mathrm{Pb}\left(\mathrm{Mg}_{1 / 3} \mathrm{Nb}_{2 / 3}\right) \mathrm{O}_{3}-\mathrm{PbTiO}_{3}(\mathrm{PMN}-32 \% \mathrm{PT})\right]$ was orientated to excite DMS lines (see Fig. 1). A region consisting of two phases indicated by two sets of lines was selected with the goal of observing the behavior of two polymorphs as a function of an applied electric field. This was achieved by orientating the sample to a noninteger $h k l$ $(0,2.08,2.39)$ to avoid the dominating Bragg reflections. The azimuthal reference was set to 110 and the azimuthal angle $(\psi)$ was set to $-10^{\circ}$. The main difficulty with multiple lines arising from multiple phases is determining which phase each line belongs to [Fig. 1(a)]. Fortuitously, the problem was simplified due to the emergence of a single locked phase [Fig. 1(b)] when a $1-\mathrm{kV} / \mathrm{mm}$ field was applied along the $00 l$ direction.

A triple intersection comprised of $\overline{2} 0 \overline{1}, 002$, and $\overline{1} \overline{1} 1$ reflections was observed from the poled sample and could be 


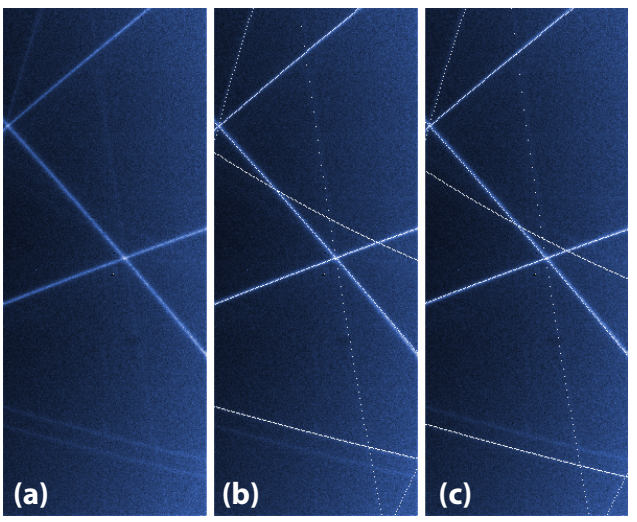

FIG. 2. (a) Two phases indicated by two sets of lines. (b) Transient monoclinic phase identified using a numerical solver minimizing two triple intersections with lattice constants [4.0039(2), 4.0206(2), 4.0312(2), 89.833(5), 90,90] and a volume of $64.895(6) \AA^{3}$. (c) Persistent monoclinic phase with lattice constants [4.0042(2), 4.0218(2), 4.0317(2), 90.158(5), 90, 90] and a volume of $64.926(6) \AA^{3}$.

used to establish the lattice parameters for the unit cell using a numerical solver. The solver calculates the stereographic projection of the DMS lines and minimizes the triangle of intersection for the projected circles. See the Supplemental Material [15]. The solver was constrained for cubic, rhombohedral, orthorhombic, and tetragonal lattices. These were then projected back on to the acquired image with the appropriate orientation matrices to superimpose the simulated triple intersections on to the triple intersections in the recorded data [Fig. 1(c)]. Only the monoclinic phase minimized by the solver from two triple intersections, comprised of the $\overline{2} \overline{1} 0, \overline{3} 03, \overline{1} 1 \overline{1}$ reflections at $7.301 \mathrm{keV}$ and the $\overline{2} \overline{1} 0, \overline{3} 03,020$ reflections at $7.309 \mathrm{keV}$, properly accounts for all of the lines [Fig. 1(d)]. The excellent agreement shown in the figure is purely the result of plotting the projection of the lines determined from the triple intersection minimization and not a fit to the data, highlighting the elegant simplicity of the triple intersection method. However, some uncertainty regarding the microscopic composition of this phase still remains. If the length scales of the tetragonal domains proposed by Jin et al. are within the coherence length of the incident beam, the DMS will be indicative of the average of the tetragonal unit cells and therefore the separate solutions cannot simply be combined to account for the detected lines. However, the DMS can still offer some insight. One of its unique features is the peak widths of in plane and out of plane reflections that can be measured simultaneously without moving the sample and without changing the footprint of the beam on the sample. If Jin's model was correct, a significant broadening in the $h k 0$ direction would be expected due to the $10-\mathrm{nm}$ separation of phases in the (110) plane. Taking into account the projection of the beam onto each of the scattering planes and the subsequent projection on to the detector plane, the peak width for the $\overline{3} 30$ reflection is $40 \%$ narrower than the 002 reflection. This is indicative of a monophasic state within the $\mathrm{x}$-ray scattering volume which is not consistent with the adaptive phase model leaving the polarization rotation model as the likely mechanism.

Working backwards from the monophasic system and following the devolution to a biphasic system, the task of assigning the DMS lines to the appropriate phase is simplified. The $002, \overline{1} \overline{1} 1, \overline{2} 0 \overline{1}, \overline{2} \overline{1} 1$, and $\overline{4} 11$ lines are common to both phases, while the $\overline{3} 30$ and $\overline{1} 2 \overline{1}$ lines are not. When using the $002, \overline{1} \overline{1} 1, \overline{2} 0 \overline{1}$ triple intersection and $002, \overline{2} \overline{1} 1, \overline{4} 11$ near triple intersection, the solver converges on two monoclinic solutions, one with a monoclinic angle greater than $90^{\circ}$ and the other less than $90^{\circ}$ (Fig. 2). While using a near intersection is not robust, the solutions in this instance provide excellent initial values for a more sophisticated fitting routine. The results were used the simulate the DMS lines which were transversely extruded to create fixed regions of interest. These transverse line intensities were then integrated along the simulated DMS lines to determine the peak centers of the real image. Using the same fixed regions of interest, a minimizer was used to navigate the parameter space to determine the set of values causing the simulated line centers to converge with those of the real data. This is done concurrently for each set of lines and is indeed confirmed in the second monoclinic phase. This methodology is more fully described in the Supplemental Material [15].

Finally, comparing the lines for the two monoclinic phases at high field with those visible at zero field for the virgin sample showed one of the monoclinic phases to be persistent. The other phase could be accounted for with tetragonal constraints. The lattice parameters for these phases at selected applied voltages are presented in Table I.

A dramatic change in the relative positions of the DMS lines is observed between 0.2 and $0.25 \mathrm{kV} / \mathrm{mm}$. This is due

TABLE I. Field induced phase transitions in PMN-PT. The letters $\mathrm{T}$ and $\mathrm{P}$ in brackets refer to the transient and persistent phases, respectively. The table shows the lattice parameters at specified electric fields. The values are given to a precision of $\pm 2 \times 10^{-4}$ for $a, b$, and $c$, and $5 \times 10^{-4}$ for $\alpha$. Where 90 is given as in integer, a constraint is implied.

\begin{tabular}{|c|c|c|c|c|c|c|c|}
\hline Field kV/mm & Phase & $a(\AA)$ & $b(\AA)$ & $c(\AA)$ & $\alpha(\operatorname{deg})$ & $\beta(\operatorname{deg})$ & $\gamma(\operatorname{deg})$ \\
\hline \multirow{2}{*}{0} & tetragonal $(\mathrm{T})$ & 4.0263 & 4.0164 & 4.0263 & 90 & 90 & 90 \\
\hline & monoclinic $(\mathrm{P})$ & 4.0056 & 4.0203 & 4.0282 & 90.215 & 90 & 90 \\
\hline \multirow{2}{*}{0.3} & monoclinic $(\mathrm{T})$ & 4.0058 & 4.0217 & 4.0266 & 89.810 & 90 & 90 \\
\hline & monoclinic $(\mathrm{P})$ & 4.0068 & 4.0202 & 4.0280 & 90.219 & 90 & 90 \\
\hline \multirow{2}{*}{1.0} & monoclinic $(\mathrm{T})$ & 4.0052 & 4.0208 & 4.0298 & 89.870 & 90 & 90 \\
\hline & monoclinic $(\mathrm{P})$ & 4.0063 & 4.0222 & 4.0264 & 90.207 & 90 & 90 \\
\hline 1.0 (monophasic after $50 \mathrm{~s}$ ) & monoclinic $(\mathrm{P})$ & 4.0057 & 4.0221 & 4.0294 & 90.197 & 90 & 90 \\
\hline
\end{tabular}




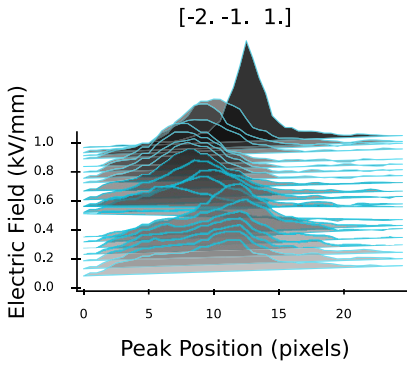

[ 0.0 .2$. .

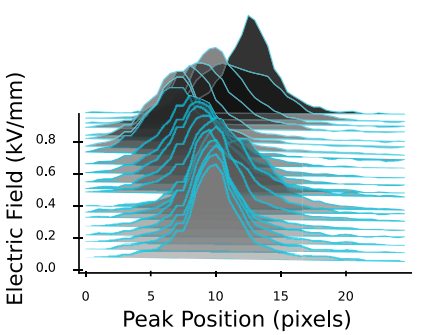

$[-2,-1,1$.

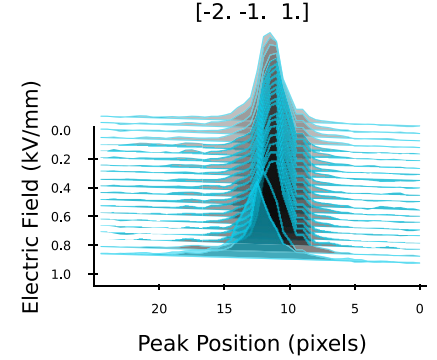

[ 0.0 .2$.

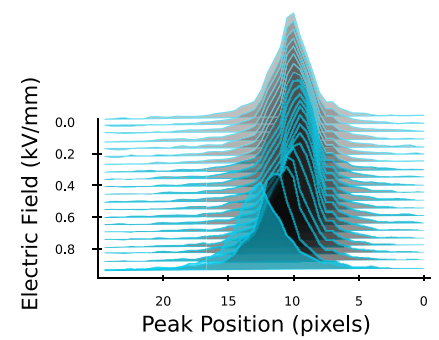

FIG. 3. Line shifts with respect to fixed regions of interest as a function of increasing (left) and decreasing (right) electric field. The blue curves on the right and the first black curves on the right are measured with the same electric field with $50 \mathrm{~s}$ between them.

to the phase change alluded to above, from tetragonal to monoclinic, resulting in a large change in the lattice parameters of $0.509 \%,-0.134 \%,-0.007 \%$, and $0.21 \%$ for $a, b, c$, and $\alpha$, respectively, over a step of only $50 \mathrm{~V} / \mathrm{mm}$. Superficially, this would suggest transient $d_{31}, d_{32}$, and $d_{33}$ coefficients of $-101830,26391$, and $1380 \mathrm{pm} / \mathrm{V}$, respectively. These values are inversely proportional to the change in electric field and are overly inflated by the absence of temporal considerations. They have been included in this form to express the scale of the changes occurring as the system jostles towards phase alignment. Finkel et al. [16] observe a change of this order of magnitude in PIN-PMN-PT under stress biased conditions. The coefficients for the persistent monoclinic phase within the same measurement are modest by comparison, 5941, -878 , and $-817 \mathrm{pm} / \mathrm{V}$. This, unsurprisingly, demonstrates that the dominant changes occur when there is a phase transition. A simple model, based on density functional theory calculations, of how the presence of the electric field changes the relative stability of the two phases, is presented in the Supplemental Material.

As the field was increased further, another abrupt change was observed at $0.95 \mathrm{kV} / \mathrm{mm}$ where the lines became sharper and better defined for the two monoclinic phases. This is clearly illustrated in Fig. 3, which shows the shift in DMS lines with respect to fixed regions of interest as a function of increasing and decreasing field. The transient monoclinic phase disappeared after $50 \mathrm{~s}$ leaving only the original monoclinic phase. However, the change in the original phase is substantial within that window of $50 \mathrm{~s}$. No reemergence of the tetragonal or transient monoclinic phases was observed as the field was reduced to $0 \mathrm{kV} / \mathrm{mm}$ nor during further repeated cycles between 0 and $1 \mathrm{kV} / \mathrm{mm}$. Once in the monophasic state the piezoelectric coefficients reduce to 342,1636 , and $994 \mathrm{pm} / \mathrm{V}$ for $d_{31}, d_{32}$, and $d_{33}$, respectively. The largest total strain, as the field is reduced, occurs between 1 and 0.6 $\mathrm{kV} / \mathrm{mm}$ (see the Supplemental Material [15]).

Conclusion. The most significant changes in the sample are observed during the initial poling of the virgin sample. Multiple phases and domains are observed, along with a significant background in the diffraction data. Lattice changes were most pronounced through the transition from a tetragonal phase to a monoclinic phase with the largest strain being in the $a$ direction with a change of $0.52 \%$. We did not observe this level of strain macroscopically in our previous work for such low electric fields [17] and it is likely a localized phenomenon facilitated by a localized stress bias as the system tends towards a single phase. Nevertheless, it does hint at what might be possible with the appropriate materials engineering and suggests the potential for reducing the material required for PET devices. The simultaneous measurement of the persistent phase shows that the strain is comparatively modest, yet it is still significantly enhanced $(0.045 \%)$ by the transition in the neighboring phase indicating that, while our results are consistent with the intrinsic polarization rotation model, extrinsic influences also play an important role in the GPE. When the system finally becomes monophasic the piezoelectric response is significantly reduced but is consistent with published strain data for PMN-PT in the range $0-1 \mathrm{kV} / \mathrm{mm}$. In summary, the results suggest that an extreme GPE is achievable in a multiphasic environment and involves a phase transition, an intermediate GPE, occurring in a multiphasic environment in which a neighboring phase goes through a transition, and the standard GPE occurring in a monophasic environment and following a polarization rotation mechanism.

We acknowledge Diamond Light Source for time on Beamline I16 under Proposal No. MT10248. We also acknowledge funding from the Office of Naval Research under the U.S. Naval Research Laboratory's Basic Research Program and Office of Naval Research Global, ONRG-NICOP Project No. N62909-18-1-2008 Electrosciences Ltd. Parts of this research work was carried out in the framework of the ADVENT project (Grant No. 16ENG06 ADVENT), which was supported by the European Metrology Programme for Innovation and Research (EMPIR). The EMPIR initiative was cofunded by the Europeans Horizon 2020 research and innovation program and the EMPIR Participating States. This work made use of ARCHER, the UK's national high-performance computing service, via the UK's HPC Materials Chemistry Consortium, which was funded by EPSRC (EP/R029431). This was also partially supported by Wave 1 of The UKRI Strategic Priorities Fund under EPSRC Grant No. EP/T001569/1, particularly the "AI for Science" theme within that grant, and The Alan Turing Institute. We acknowledge useful discussions and suggestions for interpretation of the data from K. T. Butler and J. Thiyagalingam. 
[1] I.-B. Magdu, X.-H. Liu, M. A. Kuroda, T. M. Shaw, J. Crain, P. M. Solomon, D. M. Newns, and G. J. Martyna, The piezoelectronic stress transduction switch for very large-scale integration, low voltage sensor computation, and radio frequency applications, Appl. Phys. Lett. 107, 073505 (2015).

[2] Mills, M. P., Cloud begins with coal (2013).

[3] H. Fu and R. E. Cohen, Polarization rotation mechanism for ultrahigh electromechanical response in single-crystal piezoelectrics, Nature (London) 403, 3629 (2000).

[4] B. Noheda, D. E. Cox, G. Shirane, S. E. Park, L. E. Cross, and Z. Zhong, Polarization Rotation via a Monoclinic Phase in the Piezoelectric 92\% $\mathrm{PbZn}_{1 / 3} \mathrm{Nb}_{2 / 3} \mathrm{O}_{3}-8 \% \mathrm{PbTiO}_{3}$, Phys. Rev. Lett. 86, 3891 (2001).

[5] B. Noheda, Structure and high-piezoelectricity in lead oxide solid solutions, Curr. Opin. Solid State Mater. Sci. 6, 27 (2002).

[6] D. Vanderbilt and M. H. Cohen, Monoclinic and triclinic phases in higher-order Devonshire theory, Phys. Rev. B 63, 094108 (2001).

[7] Y. M. Jin, Y. U. Wang, A. G. Khachaturyan, J. F. Li, and D. Viehland, Adaptive ferroelectric states in systems with low domain wall energy: Tetragonal microdomains, J. Appl. Phys. 94, 3629 (2003).

[8] A. Rossetti Jr., W. Zhang, and A. G. Khachaturyan, Phase coexistence near the morphotropic phase boundary in lead zirconate titanate solid solutions, Appl. Phys. Lett. 88, 072912 (2006).

[9] K. A. Schönau, L. A. Schmitt, M. Knapp, H. Fuess, R.-A. Eichel, H. Kungl, and M. J. Hoffmann, Nanodomain structure of $\mathrm{Pb}\left[\mathrm{Zr}_{1-x} \mathrm{Ti}_{x}\right] \mathrm{O}_{3}$ at its morphotropic phase boundary:
Investigations from local to average structure, Phys. Rev. B 75, 184117 (2007).

[10] H. Seemann, Zur Optik der Reflexion von Röntgenstrahlen an Kristallspaliflächen. I, Ann. Phys. 356, 391 (1916).

[11] T. Fujiwara, Memoirs of the College of Science, Kyoto Imperial University. Series A 11, 283 (1928).

[12] W. Kossel, Zur Systematik der Röntgenreflexe eines Raumgitters, Ann. Phys. 5, 417 (1936).

[13] K. Lonsdale, Divergent-beam x-ray photography of crystals, Philos. Trans. R. Soc. London 240, 219 (1947).

[14] A. G. A. Nisbet, G. Beutier, F. Fabrizi, B. Moser, and S. P. Collins, Diffuse multiple scattering, Acta Crystallogr. Sect. A 71, 20 (2015).

[15] See Supplemental Material at http://link.aps.org/supplemental/ 10.1103/PhysRevMaterials.5.L120601 for more information regarding the experimental setup, analysis methodology, and a simple model of how the presence of an electric field changes the relative stability of the two phases discussed in the text.

[16] P. Finkel, M. Staruch, A. Amin, M. Ahart, and S. E. Lofland, Simultaneous stress and field control of sustainable switching of ferroelectric phases, Sci. Rep. 5, 13770 (2015).

[17] C. Vecchini, P. Thompson, M. Stewart, A. Muiz-Piniella, S. R. C. McMitchell, J. Wooldridge, S. Lepadatu, L. Bouchenoire, S. Brown, D. Wermeille, O. Bikondoa, C. A. Lucas, T. P. A. Hase, M. Lesourd, D. Dontsov, and M. G. Cain, Simultaneous dynamic electrical and structural measurements of functional materials, Rev. Sci. Instrum. 86, 103901 (2015). 